\title{
Electric currents induced step-like resistive jumps and negative differential resistance in thin films of $\mathrm{Nd}_{0.7} \mathrm{Sr}_{0.3} \mathrm{MnO}_{3}$
}

\author{
J. F. Wang, Z. P. Wu, and J. Gao a) \\ Department of Physics, The University of Hong Kong, Pokfulam Road, Hong Kong
}

(Presented 1 November 2011; received 22 September 2011; accepted 8 November 2011; published online 2 March 2012)

\begin{abstract}
Electric-currents-induced emergent phenomena were found in microbridges of $\mathrm{Nd}_{0.7} \mathrm{Sr}_{0.3} \mathrm{MnO}_{3}$. After the samples were processed by currents of high densities, a second metal-insulator transition appeared at low temperatures. This resistance peak was very sensitive to weak currents. More salient features were the step-like resistance jumps. At temperatures near these resistance steps, negative differential resistance was observed. Interfacial effects related to electrodes could be ruled out. These effects might be due to current-enhanced inhomogeneity. (C) 2012 American Institute of Physics. [doi:10.1063/1.3675998]
\end{abstract}

\section{INTRODUCTION}

Colossal magnetoresistance manganites have been a continuing focus for decades due to their correlated physics for fundamental research and tantalizing properties for potential applications. ${ }^{1-6}$ In these materials, there are complex interconnections between different degrees of freedoms and delicate balances between rich electronic phases. It is suggested that coexistence of competing phases with close energy is at the core of colossal magnetoresistance and many other curious phenomena. An intriguing consequence of such competitions and couplings is that not only magnetic fields but also electric fields/currents and other external stimuli could modify the properties of manganites. ${ }^{7-15}$ These interplays can open a window for the understanding the physics of strong correlation and may lead to potential applications.

The effects of electric fields/currents could be dramatic and pronounced. The charge-ordered state in single crystals of $\mathrm{Pr}_{0.7} \mathrm{Ca}_{0.3} \mathrm{MnO}_{3}$ was collapsed by the electric fields/ currents. ${ }^{8}$ In such a process, the changes of resistance at low temperatures were as large as several orders. Electric currents/fields induced non-transient breakdown of charge-ordered state was found in single crystals of $\mathrm{Nd}_{0.5} \mathrm{Sr}_{0.5} \mathrm{MnO}_{3} .{ }^{9}$ In thin films of $\mathrm{La}_{1-\mathrm{x}} \mathrm{Ca}_{\mathrm{x}} \mathrm{MnO}_{3}(\mathrm{x}=0.2$, $0.3)$, highly resistive metastable states, which exhibited huge electroresistance, were excited by currents of high densities. ${ }^{10}$ Even asymmetric conduction could be induced by large currents from uniform virgin states. ${ }^{11}$ By controlling the current pulse pair, reversible resistance switching was achieved in bulk $\mathrm{La}_{0.225} \mathrm{Pr}_{0.4} \mathrm{Ca}_{0.375} \mathrm{MnO}_{3} .{ }^{12}$ For thin films of $\mathrm{Pr}_{0.7} \mathrm{Sr}_{0.3} \mathrm{MnO}_{3}$, current processing yielded low-temperature persistent conductivity. ${ }^{13}$ In this paper, we report currentinduced emergent properties, including metal-insulator transition, resistance steps and negative differential resistance, in thin films of $\mathrm{Nd}_{0.7} \mathrm{Sr}_{0.3} \mathrm{MnO}_{3}$ (NSMO).

\footnotetext{
a) Author to whom correspondence should be addressed. Electronic mail: jugao@hku.hk.
}

\section{EXPERIMENTAL}

Thin films of NSMO on (001) oriented $\mathrm{SrTiO}_{3}$ (STO) were fabricated using pulsed laser ablation. ${ }^{16}$ During growth, the substrate holder was kept at $\sim 720^{\circ} \mathrm{C}$. Subsequent to deposition, the samples were annealed in 1 bar oxygen at grown temperature for $30 \mathrm{~min}$. As measured with a Dektak stylus profiler, the thickness is $\sim 60 \mathrm{~nm}$. The films were patterned into microbridges with a width of $\sim 20 \mu \mathrm{m}$ and a length of $\sim 100 \mu \mathrm{m}$ (see the inset of Fig. 1) using photolithography. Silver (Ag) electrodes were deposited by thermal evaporation. The contacts between Ag and NSMO are ohmic. Four-probe method was adopted to measure the transport properties.

\section{RESULTS AND DISCUSSIONS}

Figure 1 shows the typical $x$-ray diffraction (XRD) pattern of NSMO on STO. There are no extra peaks other than (001) peaks of NSMO and STO. As calculated from the strongest reflection peak $[\mathrm{NSMO}(002)]$, the out-of-plane lattice constant of the NSMO film is $\mathrm{c} \sim 3.830 \AA$. A distinct transition from metal to insulator occurs at $\sim 160 \mathrm{~K}\left(\mathrm{~T}_{\mathrm{P}}\right)$ and the resistivity at the peak is about $1 \Omega \mathrm{cm}$. These values are consistent with those reported in the literature. ${ }^{17}$ Increasing the measurement current from $1 \mu \mathrm{A}$ to $50 \mu \mathrm{A}$ did not influence the position of $T_{P}$ and the value of peak resistance. This implies that a virgin state of NSMO is not sensitive to weak currents.

These microbridges exhibited interesting features after processed with currents of a high density (I $\sim 5 \mathrm{~mA}$ and $\mathrm{J}_{\mathrm{C}} \sim 4 \times 10^{5} \mathrm{~A} / \mathrm{cm}^{2}$ ) at room temperature. Figure 2(a) depicts the evolution of current-excited state with processing period. There are two peaks in $\mathrm{R}(\mathrm{T})$ curves for the metastable states induced with currents of short durations, e.g., $\delta \mathrm{t}=5$ and $7 \mathrm{~min}$. One peak is correlated with that in pristine state, and the other is located at a lower temperature. Similar to that in the initial state, the high-temperature peaks are not susceptible to weak currents. On contrary, the low-temperature peaks may be strongly influenced by currents, as shown in Fig. 2(b). With the increase of measuring current, the resistance around the low-temperature peak was greatly reduced. 


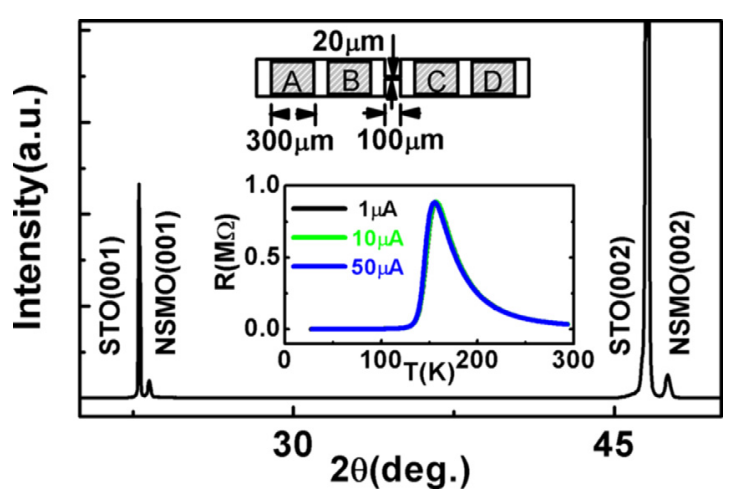

FIG. 1. (Color online) XRD pattern ( $\theta-2 \theta$ scan) of NSMO/STO. The insets: the layout of the microbridge (upper) and $\mathrm{R}(\mathrm{T})$ curves recorded with different currents (lower)

When the duration was extended to $10 \mathrm{~min}$, the resistance of the excited state showed a monotonous increase with decreasing temperatures. There was no obvious change of conducting behavior in the whole temperature range but only an anomaly around the transition temperature of the virgin state. Intriguingly, this state did not show any susceptibility to weak currents [Fig. 2(c)]. Therefore, there was a clear evolution of low-temperature state during current processing. Initially, the low-temperature state was dominated by metallic phases. With the increase of processing durations, the insulating portions increased. The low-temperature resistance peaks manifest the competition between metallic phases and insulating ones. Finally, the portion of insulating phases was much larger than that of metallic ones. At the same time, the sensitivity to weak currents first increased and then decreased.

To see whether the interfacial effects are the origins, we measured two-lead $\mathrm{R}(\mathrm{T})$ and $\mathrm{I}(\mathrm{V})$ curves (not shown) between electrodes $A(C)$ and $B(D)$ (see the inset of Fig. 1) prior to and after each current processing. There was no substantial change, implying the interfacial effects are not relevant. Thus
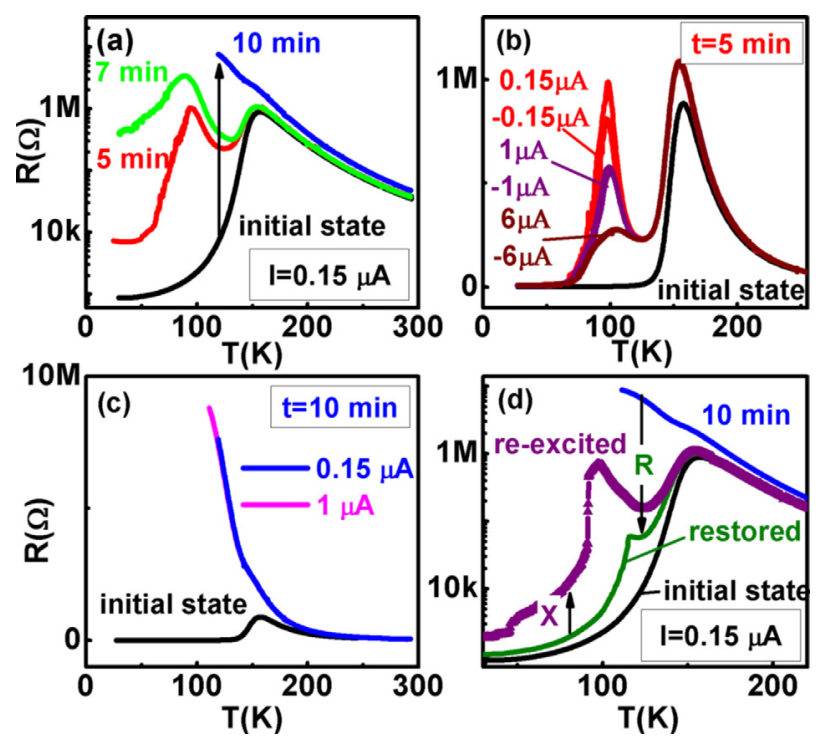

FIG. 2. (Color online) (a) $\mathrm{R}(\mathrm{T})$ curves measured with a current of $0.15 \mu \mathrm{A}$ subsequent to current processing (I $\sim 5 \mathrm{~mA}$ and $\mathrm{J}_{\mathrm{C}} \sim 4 \times 10^{5} \mathrm{~A} / \mathrm{cm}^{2}$ ) with durations of 5, 7, and $10 \mathrm{~min}$; (b), (c) R-T curves for current-excited states measured with different currents; (d) the restoration (process R) and re-excitation (process $\mathrm{X}$ ) induced by a large negative current $(\mathrm{I} \sim-5 \mathrm{~mA})$. the changes should take place within the microbridge. The electric fields/currents-induced effects have not been wellunderstood. The reported consequences of electric fields/currents are diverse. ${ }^{8-15}$ Both decrease and increase of resistance were observed. To understand such effects, it may be useful to note the features of energy landscape. In manganites, different electronic ground states are energetically comparable to each other and constitute a multivalley energy landscape., Various electronic states may coexist in a single chemical phase. Stimuli like electric fields/currents might drive the system from one energy valley to another. At the same time, the transport properties would be modified.

The samples could be partly restored by negative currents $(\mathrm{I} \sim-5 \mathrm{~mA})$ with a proper duration $(\sim 1 \mathrm{~min})$. Here, the direction of the processing current was defined as the positive one. As displayed in Fig. 2(d), the restored state was very close to the initial state except a kink at the lower shoulder of the resistance peak. Extending the duration of negative current re-excited the low-temperature peak. The re-excited resistance peak was also sensitive to small currents. A remarkable feature is the step-like features at the lower shoulder. For $\mathrm{I}=0.15 \mu \mathrm{A}$, after several steps, the resistance changed about one order. For larger currents, the discrete steps were also evident though the magnitudes were smaller [see Fig. 3(a)].

To get some insights, current-voltage curves were also recorded for the re-excited state. At high temperatures, only linear relations (not shown) were observed. This is consistent
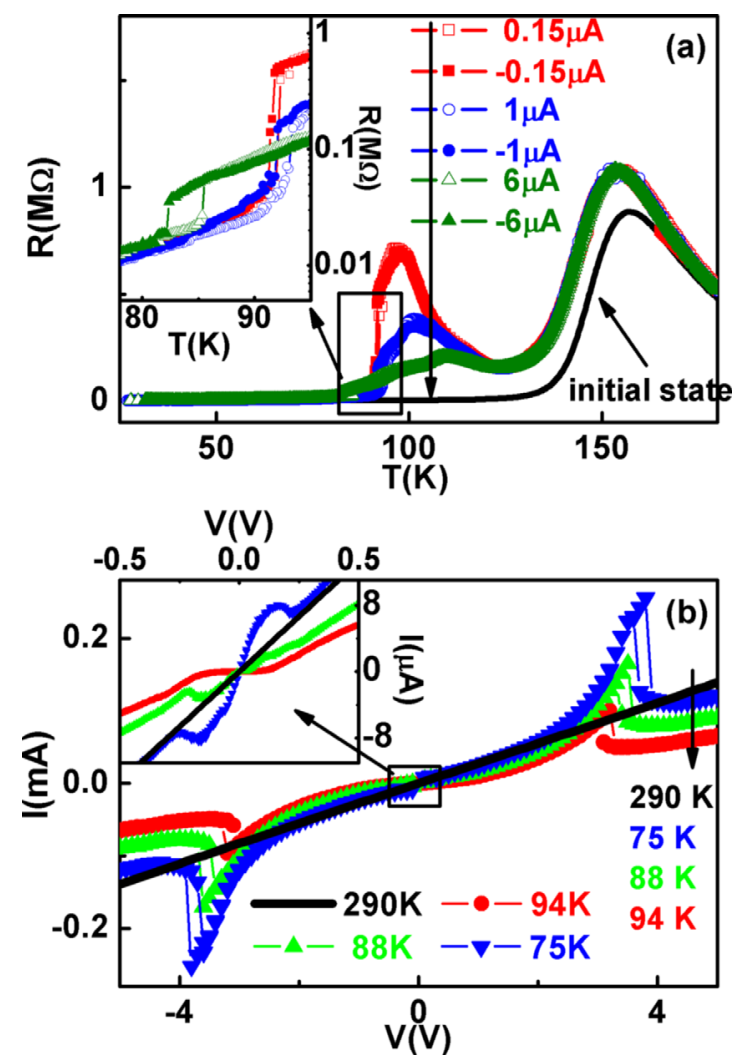

FIG. 3. (Color online) (a) Temperature dependence of resistance measured with different currents for the re-excited state. The inset: magnified view around the resistance steps; (b) current-voltage curves at temperatures around the resistance steps. For reference, the I(V) curve at $290 \mathrm{~K}$ is also shown. The inset: magnified view for low bias voltages. 


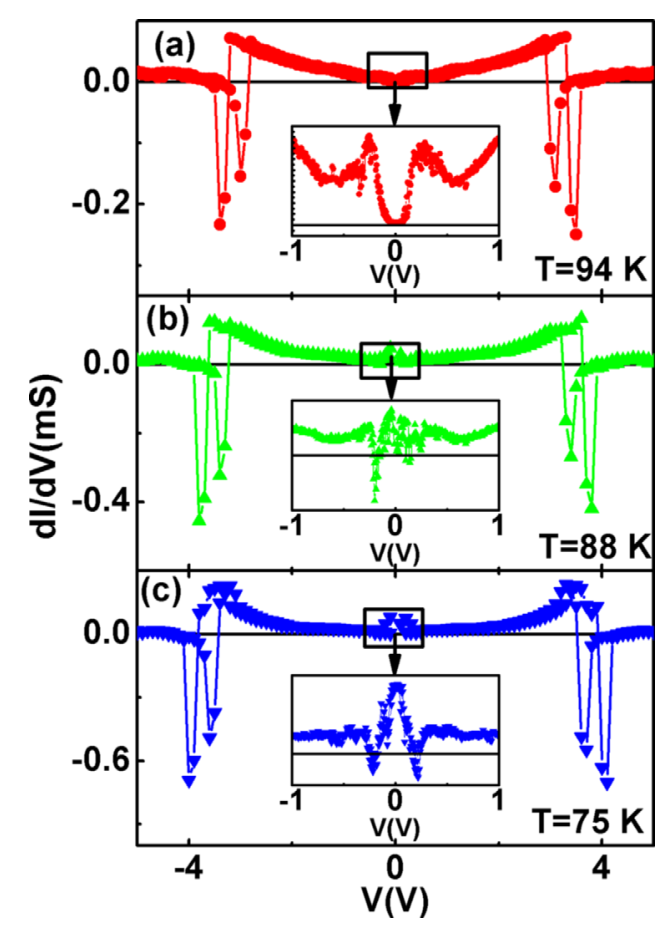

FIG. 4. (Color online) Differential conductance $\mathrm{dI} / \mathrm{dV}$ as a function of voltage $\mathrm{V}$ (from $-5 \mathrm{~V}$ to $5 \mathrm{~V}$ ) at $94 \mathrm{~K}$ (a), $88 \mathrm{~K}$ (b), and $75 \mathrm{~K}$ (c). The insets: magnified views for low bias voltages.

with the features in R-T curves shown in Fig. 3(a), where the $\mathrm{R}$ - $\mathrm{T}$ curves above $125 \mathrm{~K}$ do not change as the current varies. At lower temperatures, the isothermal current-voltage curves are complex and intriguing. Displayed in Fig. 3(b) are current-voltage characteristics measured by sweeping voltages in the vicinal temperatures of resistance steps. At a first glance, these I-V curves are similar. At $\mathrm{V} \sim \pm 4 \mathrm{~V}$, characteristics of negative differential resistance (NDR) appear. As can be seen from Fig. 4, in this region, there are clear negative peaks in the $\mathrm{dI} / \mathrm{dV}-\mathrm{V}$ plots. However, considerable differences can be found from the magnified views at lowvoltage region [see the inset of Fig. 3(b) and the insets of Fig. 4]. At $88 \mathrm{~K}$ and $75 \mathrm{~K}$, when $\mathrm{V} \sim \pm 0.2 \mathrm{~V}$, there are also features of NDR. On contrary, at $94 \mathrm{~K}$, the current changes smoothly in this region of voltages. It is noted that when NDR appears, for one current, there could be three possible resistance states. Therefore, there should be correlations between the resistance steps in Fig. 3(a) and NDR in Fig. 3(b).

For the same reason as above, the interfacial effects should not be relevant to the resistance steps and NDR. These phenomena reminded us of those observed in spatially confined films of ( $\mathrm{La}, \mathrm{Pr}, \mathrm{Ca}) \mathrm{MnO}_{3}$ and (Pr, Ca, $\mathrm{Sr}) \mathrm{MnO}_{3},{ }^{18-21}$ two well-known systems of phase separation. By considering the effects of spatial confinement, reemergent metal-insulator transition and negative differential resistance were qualitatively reproduced using a resistive network model. ${ }^{18,21,22}$ It should be reasonable to speculate that multiphase coexistence and spatial confinement are also key ingredients to understand our results. As discussed above, stimuli like large electric fields/currents might drive the system from one energy valley to another. ${ }^{5,6}$ It is possible that after current processing there are finite conduction filaments through the microbridge in certain temperature range. The current densities in these conducting filaments are much larger than those in other parts. Severe heating in these filaments could consequently shut off the conduction path. At the points the conduction filaments are created/annihilated, resistive steps and negative differential resistance may appear. ${ }^{19-22}$ The exact origins of these current-induced emergent properties need a further study.

\section{CONCLUSIONS}

In brief words, the current-induced effects in epitaxial thin films of $\mathrm{Nd}_{0.7} \mathrm{Sr}_{0.3} \mathrm{MnO}_{3}$ were studied. Initially, the lowtemperature state was dominated by metallic phases. The insulating portions increased while duration of current processing was extended. As a consequence, an additional resistance peak, which was very sensitive to weak currents, appeared in the $\mathrm{R}(\mathrm{T})$ curve. The samples could be partly restored and re-excited by negative currents with proper durations. For the re-excited state, discrete resistance steps in the $R(T)$ curves were observed. At temperatures around these steps, there were negative differential resistance and complex electroresistance. These phenomena are probably due to current-enhanced inhomogeneity in the microbridges.

\section{ACKNOWLEDGMENTS}

This work was supported by a grant of the Research Grant Council of Hong Kong (Project No. HKU 702409 P) and a Seed Funding from the University of Hong Kong.

${ }^{1}$ R. von Helmolt, J. Wecker, B. Holzapfel, L. Schultz, and K. Samwer, Phys. Rev. Lett. 71, 2331 (1993).

${ }^{2}$ S. Jin, T. H. Tiefel, M. McCormack, R. A. Fastnacht, R. Ramesh, and L. H. Chen, Science 264, 413 (1994).

${ }^{3}$ Y. Tokura, A. Urushibara, Y. Moritomo, T. Arima, A. Asamitsu, G. Kido, and N. Furukawa, J. Phys. Soc. Jpn. 63, 3931 (1994).

${ }^{4}$ M. B. Salamon and M. Jaime, Rev. Mod. Phys. 73, 583 (2001).

${ }^{5}$ E. Dagotto, T. Hotta, and A. Moreo, Phys. Rep. 344, 1 (2001).

${ }^{6}$ Y. Tokura, Rep. Prog. Phys. 69, 797 (2006).

${ }^{7}$ T. Mori, K. Ogawa, K. Yoshida, K. Miyano, Y. Tomioka, and Y. Tokura, J. Phys. Soc. Jpn. 66, 3570 (1997).

${ }^{8}$ A. Asamitsu, Y. Tomioka, H. Kuwahara, and Y. Tokura, Nature 388, 50 (1997).

${ }^{9}$ T. Mori, Phys. Rev. B 58, 12543 (1998).

${ }^{10}$ J. Gao and F. X. Hu, Appl. Phys. Lett. 86, 092504 (2005).

${ }^{11}$ F. X. Hu and J. Gao, Appl. Phys. Lett. 87, 152504 (2005).

${ }^{12}$ Z. B. Yan, K. F. Wang, S. Z. Li, S. J. Luo, and J. M. Liu, Appl. Phys. Lett. 95, 143502 (2009).

${ }^{13}$ J. F. Wang and J. Gao, J. Appl. Phys. 109, 07 d701 (2011).

${ }^{14}$ V. Markovich, G. Jung, Y. Yuzhelevski, G. Gorodetsky, A. Szewczyk, M. Gutowska, D. A. Shulyatev, and Y. M. Mukovskii, Phys. Rev. B 70, 064414 (2004).

${ }^{15}$ T. Wu, S. B. Ogale, J. E. Garrison, B. Nagaraj, A. Biswas, Z. Chen, R. L. Greene, R. Ramesh, T. Venkatesan, and A. J. Millis, Phys. Rev. Lett. 86, 5998 (2001).

${ }^{16}$ J. Gao, S. Y. Dai, and T. K. Li, Phys. Rev. B 67, 153403 (2003).

${ }^{17}$ A. Barman and G. Koren, Appl. Phys. Lett. 77, 1674 (2000).

${ }^{18}$ T. Z. Ward, S. Liang, K. Fuchigami, L. F. Yin, E. Dagotto, E. W. Plummer, and J. Shen, Phys. Rev. Lett. 100, 247204 (2008).

${ }^{19}$ H.-Y. Zhai, J. X. Ma, D. T. Gillaspie, X. G. Zhang, T. Z. Ward, E. W. Plummer, and J. Shen, Phys. Rev. Lett. 97, 167201 (2006).

${ }^{20}$ T. Wu and J. F. Mitchell, Appl. Phys. Lett. 86, 252505 (2005).

${ }^{21}$ T. Wu and J. F. Mitchell, Phys. Rev. B 74, 214423 (2006).

${ }^{22}$ J. C. Wu, H. Sun, H. X. Da, and Z. Y. Li, Appl. Phys. Lett. 91, 102501 (2007). 